\title{
Can examination of tissue stained with Oil red O be postponed up to three months?
}

\author{
Simone Dorthea Christoffersen, stud. med., Jørgen Lange Thomsen, MD
}

Institute of Forensic Medicine, University of Southern Denmark

\begin{abstract}
Introduction: As far as we know, there are no known studies on the durability of frozen tissue stained with Oil Red O. The purpose of this study was to examine if the lipid drops in Oil Red O stained tissue keep the original position and color over time ( 3 months). Further we examined if storage position of the stained tissue makes a difference. Method: We used ten frozen kidney sections stained with Oil Red $\mathrm{O}$. Half of the samples were stored vertically and the other half horizontally, and photos of the same areas were taken within the first 24 hours after staining, and then after 48 hours, 72 hours, 7 days, 14 days, 1 month, 2 months and 3 months respectively. Results and conclusion: No changes in position of the lipids were observed. The color of the staining faded somewhat over time, but it was still possible to distinguish the positive sites from the negative.
\end{abstract}

Keywords:

Oil Red O, Staining, Kidney, Stability

\section{INTRODUCTION}

Oil red $\mathrm{O}$ (or Oil Red $4 \mathrm{~B}$ ) is a lysochrome used in histochemistry for the staining of neutral triglycerides. Lipids in frozen tissue stained with Oil Red $O$ will appear as red drops, making them easy to distinguish from other tissue components, which will appear blue because of the nuclear staining. Oil Red $O$ staining is widely used for example for quantifying liver steatosis [1]. In forensic pathology Oil Red $\mathrm{O}$ is used for detecting lipids in the proximal tubules of the kidneys as a diagnostic marker for diabetes [2] and alcoholic ketoacidosis [3]. Oil Red O staining is also suggested to be a diagnostic marker for death due to hypothermia $[4,5]$, and for detection of fat emboli in the lungs.

As far as we know, there are no studies on the durability of frozen tissue Oil Red $O$ staining. It has been suggested, that tissue stained with Oil Red O must be examined within 24 hours, as large drops of lipid may allegedly derange and float together because of the smaller surface tension of the lysocrom organic solvent.

The purpose of this study was to examine if the lipid drops in Oil Red O stained tissue keep the original position and color over time (3 months). Furthermore we examined if the storage position of the stained tissue makes a difference.

\section{METHOD}

Kidney samples with lipid from ten cases were obtained at the Department of Forensic Medicine, University of Copenhagen. The tissue was kept at minus 80 degrees Celsius until it was cut and mounted on pre-cleaned Superfrost Plus microscope slides. Immediately after mounting, the sections were frozen again. The sections were transported to Odense by train ( $1 \frac{1 / 2}{2}$ hours) and were put back in a freezer (minus 20 degrees) immediately after arrival.
The sections were all stained at the Institute of Forensic Medicine, University of Southern Denmark. The procedure was: First staining of the nuclei with hematoxylin Carrazi, then washing in water and $77 \%$ ethanol, and after this the sections were stained with the Oil red $\mathrm{O}$ solution for 15 minutes. Finally the sections were quickly washed in $77 \%$ ethanol and in water again for at least 10 minutes, and the sections were mounted in aquamount [6].

Each sample was examined and photos were taken at $\times 40, x 100$ and $\times 200$ magnification within the first 24 hour after staining. Half of the samples were stored horizontally, and the other half stored vertically in small plastic boxes. All the samples were examined at the same time and stored darkly in the same area with a constant room temperature. The same microscope was used after 48 hours, 72 hours, 7 days, 14 days, 1 month, 2 months and 3 months respectively. Photos of the same part of each sample were taken each time. Both positive and negative sites of the sample were documented visually.

At the end the photos were compared and it was determined whether lipids in each sample had moved from their original position and if there was a difference between the samples stored horizontally and the samples stored vertically. Furthermore it was determined if there were any changes in the color in the positive sites. All comparisons were done with the naked eye by two observers (the authors).

\section{RESULTS}

After 3 months it was concluded, that the lipid stains in the positive samples did not move from their original position. It was observed, that the staining began to fade after two weeks, and that the fading was more visible after three months although at this time it was still possible to distinguish the positive sites from the negative (Fig. 1). We concluded that there was no visible difference between the samples stored in vertical and horizontal positions respectively. 

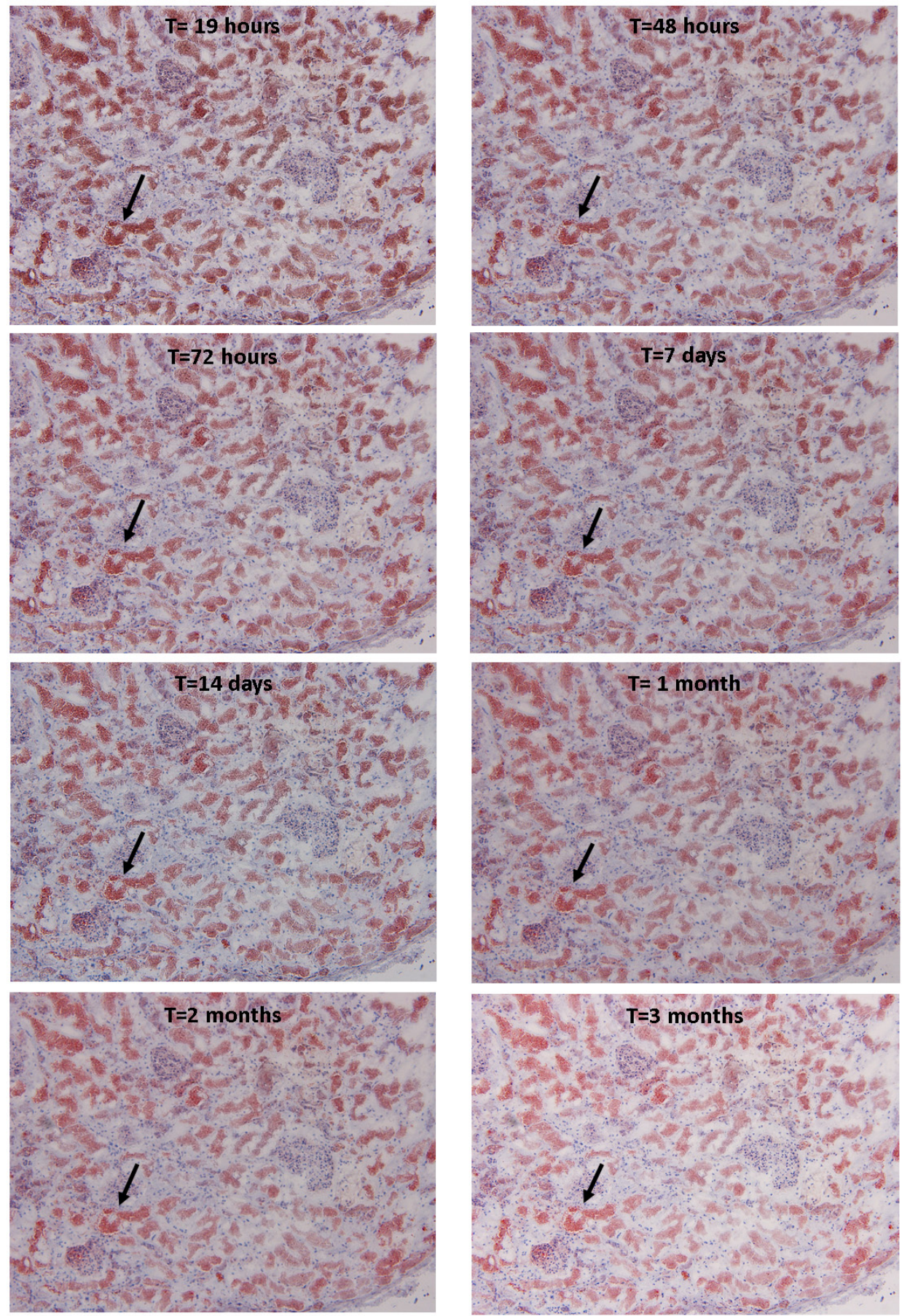

Figure 1. A section stained with Oil Red $O$ and followed over time. Section stored vertically. Original magnification $\times 100$. Arrow: stained tubule.

\section{DISCUSSION}

It is believed, that Oil red O stained tissue must be examined within 24 hours because of the risk of the lipid deranging over time. In this study we found that examination of tissue stained with Oil red $\mathrm{O}$ can be postponed up to three months, without major changes in the tissue. We therefore find no evidence to support the above-mentioned assertion.

Although the sample size in this study is fairly small we do not suspect, that a larger sample size would have changed our conclusion.

\section{CONCLUSION}

In a busy working day, microscopy of Oil red O stained frozen sections can, if necessary, be postponed up to three months, although some fading of the sections may be apparent.

\section{DISCLOSURES}

No conflicts of interest, financial or otherwise, are declared by the authors. 


\section{REFERENCES}

[1] Levene AP, Kudo H, Thursz MR, Anstee QM, Goldin RD. Is Oil Red-O Staining and Digital Image Analysis the Gold Standard for Quantifying Steatosis in the Liver? Hepatology. 2010;51(5):1859-.

[2] Thomsen JL, Hansen TP. Lipids in the proximal tubules of the kidney in diabetic coma. The American journal of forensic medicine and pathology. 2000;21(4):416-8.

[3] Parai JL, Kodikara S, Milroy CM, Pollanen MS. Alcoholism and the Armanni-Ebstein lesion. Forensic science, medicine, and pathology. 2012;8(1):19-22.
[4] Hirvonen J. Necropsy findings in fatal hypothermia cases. Forensic Science. 1976;8(0):155-64.

[5] Preuss J, Dettmeyer R, Lignitz E, Madea B. Fatty degeneration in renal tubule epithelium in accidental hypothermia victims. Forensic science international. 2004;141(2-3):131-5.

[6] Pearse AGE. Histochemistry Theoretical and Applied. Fourth ed1985. 837 p. 